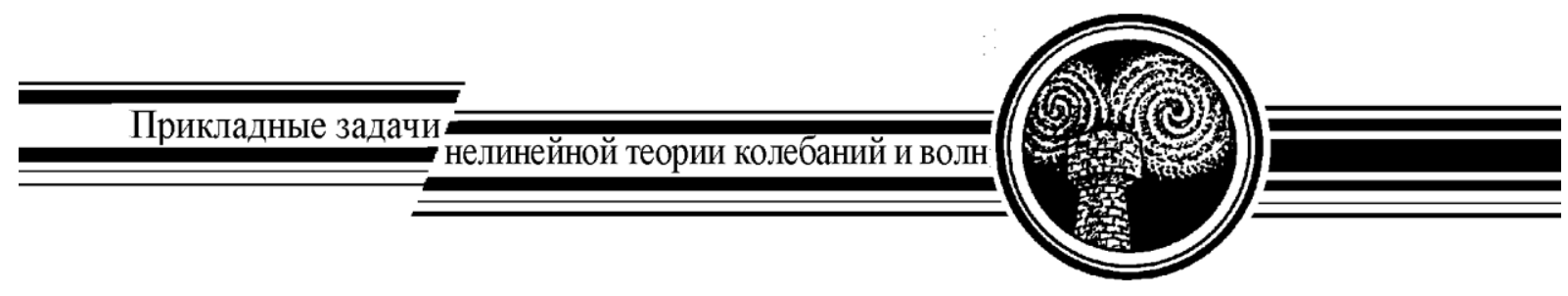

УДК $535.015 ; 535.14 ; 535.530 ; 537.86$

https://doi.org/10.18500/0869-6632-2019-27-3-61-72

\title{
Влияние запаздывания на динамику оптического параметрического осциллятора с внутрирезонаторной накачкой полупроводниковым дисковым лазером
}

\author{
Л. А. Кочкуров ${ }^{1}$, М. И. Балакин ${ }^{1}$, П. В. Купиов ${ }^{1,2}$, Ю. А. Морозов ${ }^{2}$ \\ ${ }^{1}$ Саратовский государственный технический университет им. Гагарина Ю.А. \\ Россия, 410054 Саратов, ул. Политехническая, 77 \\ ${ }^{2}$ Саратовский филиал Института радиотехники и электроники \\ им. В.А. Котельникова РАН \\ Россия, 410019 Саратов, ул. Зеленая, 38 \\ E-mail: 1kochkurov@gmail.com, balakinmaxim@gmail.com, p.kuptsov@rambler.ru,yuri.mor@rambler.ru \\ Автор для переписки Кочкуров Леонид Алексеевич, lkochkurov@gmail.com \\ Поступила в редакиию 28.03.2019; принята к публикации 15.05.2019
}

Тема. Исследована динамика численной модели нелинейного оптического взаимодействия в резонаторе полупроводникового дискового лазера с учетом временного запаздывания. Проанализированы условия самовозбуждения колебаний, режимы стационарной генерации и их устойчивость. Методы. Для анализа устойчивости стационарной генерации был использован пакет DDE-Biftool. Анализ динамических режимов более высокой размерности проводился с помощью численного интегрирования, построения фазовых портретов, спектров и расчета показателей Ляпунова. Результаты. Проведено численное моделирование динамики излучения в области неустойчивости стационарного состояния. Показано, что неустойчивость носит квазигармонический характер только вблизи бифуркации Андронова-Хопфа, и быстро принимает квазипериодический характер при вариации управляющих параметров. Изучены переходные процессы в системе. Обсуждение. Полученные результаты могут быть использованы для оптимизации параметров лазерных генераторов в устройствах спектроскопии высокого разрешения.

Ключевые слова: оптический параметрический осциллятор, полупроводниковый дисковый лазер, нелинейно-оптическое взаимодействие, динамическая система с запаздыванием.

Образец цитирования: Кочкуров Л.А., Балакин М.И., Купцов П.В., Морозов Ю.А. Влияние запаздывания на динамику оптического параметрического осциллятора с внутрирезонаторной накачкой полупроводниковым дисковым лазером// Известия вузов. ПНД. 2019. Т. 27, № 3. С. 61-72. https://doi.org/10.18500/0869-6632-2019-27-3-61-72

Финансовая поддержка. Работа выполнена при поддержке грантов РФФИ № 18-32-01028-мол_а и 18-08-00599-а, а также в рамках государственного задания ИРЭ им. В.А. Котельникова РАН.

(C) Кочкуров Л.А., Балакин М.И., Купцов П.В., Морозов Ю.А.

Известия вузов. ПНД, 2019, т. 27, № 3 


\title{
Impact of time delay on the dynamics of optical parametric oscillator with intra-cavity pumping by semiconductor disk laser
}

\author{
L.A. Kochkurov ${ }^{1}$, M.I. Balakin ${ }^{1}$, P.V. Kuptsov ${ }^{1,2}$, Yu.A. Morozov ${ }^{2}$ \\ ${ }^{1}$ Yuri Gagarin State Technical University of Saratov \\ 77, Politechnicheskaya st., 410054 Saratov, Russia \\ ${ }^{2}$ Kotelnikov Institute of RadioEngineering and Electronics of RAS, Saratov Branch \\ 38, Zelenaya st., 410019 Saratov, Russia \\ E-mail: 1kochkurov@gmail.com, balakinmaxim@gmail.com,p.kuptsov@rambler.ru, yuri.mor@rambler.ru \\ Correspondence should be addressed to Kochkurov Leonid A., 1kochkurov@gmail.com \\ Received 28.03.2019; accepted for publication 15.05.2019
}

\begin{abstract}
Theme. The dynamics of a nonlinear numerical model of a nonlinear optical interaction in the semiconductor disk laser resonator under influence of the time delay is investigated. The conditions of self-excitation, stationary generation modes and their stability are studied. Methods. The analysis of stationary generation stability was performed with DDEBiftool package. Analysis of higher dimensional regimes was performed using numerical integration, construction of phase portraits, spectra and calculation of Lyapunov exponents. Results. A numerical simulation of the dynamics in the region of steady state instability shown, that the instability is quasi-harmonic only in the vicinity of Andronov-Hopf bifurcation, and quickly turns into quasi-periodic instability with variation of control parameters. Transient dynamics is studied. Discussion. The results can be used for optimization of laser generator parameters in high resolution spectroscopy devices.
\end{abstract}

Key words: optical parametric oscillator, semiconductor disk laser, nonlinear optical interaction, time delayed systems.

Reference: Kochkurov L.A., Balakin M.I., Kuptsov P.V., Morozov Yu.A. Impact of time delay on the dynamics of optical parametric oscillator with intra-cavity pumping by semiconductor disk laser. Izvestiya VUZ, Applied Nonlinear Dynamics, 2019, vol. 27, no. 3, pp. 61-72. https://doi.org/10.18500/0869-6632-2019-27-3-61-72

Acknowledgements. This work was supported by the Russian Foundation for Basic Research (projects nо. 18-32-01028-мол_а and no. 18-08-00599 a), and was carried out at IREE RAS in the framework of the state task.

\section{Введение}

Когерентные источники непрерывного излучения в среднем и дальнем инфракрасном диапазонах находят широкое применение во многих прикладных областях, среди которых одной из самых значимых является спектроскопия высокого разрешения. Вследствие этого, исследование рабочих характеристик генераторов разностной частоты (ГРЧ) и оптических параметрических осцилляторов (ОПО) становится важной задачей [1]. Некоторые реализации таких источников могут быть достаточно просты для изготовления, компактны и удобны в применении $[2,3]$. Компактность и эффективность этих устройств может быть достигнута за счет использования схемы с внутрирезонаторным нелинейным преобразованием частоты. Пример подобного рода был, в частности, представлен в работе [4]. Однако предложенный в этой работе подход не позволяет реализовать условия, при которых длины волн сигнального и накачивающего излучений были бы достаточно близки друг другу для обеспечения холостого излучения с длиной волны порядка десятков микрометров. В работе [2] было предложено использовать общий резонатор как для волны накачки, так и для сигнальной волны, вследствие чего эта особенность была устранена. Кроме того, как было теоретически показано в работе [3], использование данного подхода позволяет создать как внутрирезонаторный ГРЧ, так и внутрирезонаторный ОПО.

Одним из факторов, определяющих возможность применимости таких устройств для прикладных задач, является устойчивость их стационарного режима генерации. Для анализа режимов генерации и их устойчивости используются математические модели, формализующие поведение динамических систем. В большинстве математических моделей внутрирезонаторных 
генераторов с нелинейным частотным преобразованием применяется метод разложения поля по нормальным модам резонатора [3,5-9]. В приближении одной моды (или для несфазированных мод) такой метод не позволяет изучать возможные быстрые (на масштабе времени обхода по резонатору) колебания амплитуд оптических полей.

Для анализа быстрых амплитудных осцилляций (которые могут явиться причиной нестабильности стационарного режима) мы используем модель лазера со связанными резонаторами, один из которых является внешним по отношению к другому [10-13]. Внутренний резонатор образован полупроводниковым брэгговским зеркалом (Б3) и излучающей поверхностью полупроводникового чипа. Внешний резонатор ограничивается выходным сферическим зеркалом с высоким коэффициентом отражения, с одной стороны, и упомянутым Б3 с другой. Было показано, что такой лазер может рассматриваться как динамическая система с запаздыванием, демонстрирующая сложное поведение [13]. Подобная модель кардинально отличается от широко известной модели Ланга-Кобаяши [14], в которой учитываются лишь слабые отражения от внешнего зеркала.

Недавно в работе [15] нами было выполнено развитие модели [13], позволяющее учесть влияние нелинейно-оптического взаимодействия в резонаторе полупроводникового дискового лазера на характеристики его излучения. В данной работе мы проводим углубленный анализ динамики излучения внутрирезонаторного ОПО.

\section{1. Динамические уравнения оптического параметрического осциллятора}

Устройство однорезонаторного ОПО, построенного на основе нелинейно-оптического взаимодействия в резонаторе полупроводникового дискового лазера (ПДЛ), схематически показано на рис. 1 [2,3]. Колебательная система выполнена по трехзеркальной схеме. Внешний резонатор, ограниченный вогнутым зеркалом с высоким коэффициентом отражения, связан с внутренним резонатором, образованным распределенным двухполосным БЗ и выходным торцом полупроводникового чипа. Внутренний резонатор по сути является активным зеркалом. Волна накачки (1.98 мкм) и сигнала (2.25 мкм) являются модами общего внешнего резонатора. Предполагается, что трехволновое нелинейно-оптическое взаимодействие осуществляется в нелинейном кристалле с регулярной доменной структурой на основе GaAs. Выходное сферическое зеркало является прозрачным для холостого излучения с длиной волны 16.5 мкм. Для обеспечения высокого коэффициента отражения активного зеркала на волнах накачки и сигнала было использовано двух-

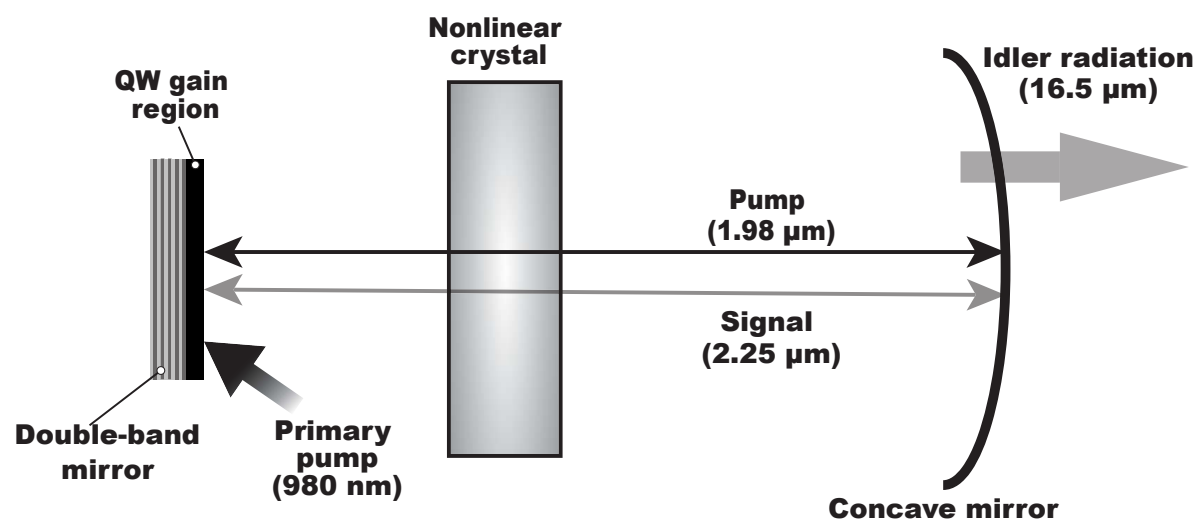

Рис. 1. Схема однорезонаторного оптического параметрического осциллятора

Fig. 1. Schematic setup of a single cavity optical parametric oscillator (OPO) 
полосное Б3 [16]. Первичная оптическая накачка активной области осуществляется с помощью диодного лазера с длиной волны 980 нм.

Устройство активного зеркала существенным образом не отличается от устройства этого элемента обычного ПДЛ: это зеркало содержит квантовые ямы на основе $\mathrm{Ga}_{0.74} \mathrm{In}_{0.26} \mathrm{Sb}$, расположенные в узлах стоячей волны накачки для обеспечения максимально возможного усиления [17].

Построение модели внутрирезонаторного ОПО [15] основано на рассмотрении отражений в колебательной системе, состоящей из связанных резонаторов (см. рис. 3 из [15] и последующие пояснения). В соответствии с подходом $[12,13]$, уравнения математической модели формулируются для переменных, относящихся к внутреннему резонатору (область длиной $L_{i n}$ на этом рисунке).

Следуя $[13,15]$, можно получить скоростные (балансные) уравнения, представляющие динамическую систему с запаздыванием, обусловленным задержкой сигнала при обходе по внешнему резонатору:

$$
\begin{aligned}
& \dot{a}_{1}=\eta\left[\left(G_{1}-1\right)+\frac{1}{T}\left(\frac{a_{1 \tau}}{a_{1}}-1\right)-\frac{\delta}{T}\left(a_{2}+a_{2 \tau}\right)\right] a_{1}, \\
& \dot{a}_{2}=\eta\left[-1+\frac{1}{T}\left(\frac{a_{2 \tau}}{a_{2}}-1\right)+\frac{\delta}{T}\left(a_{1}+a_{1 \tau}\right)\right] a_{2}, \\
& \dot{v}_{1}=\sigma-v_{1}-G_{1} a_{1} .
\end{aligned}
$$

Здесь число фотонов $a_{i}$ и число носителей $v_{1}$ нормализованы на $a_{10}$ и $v_{1 \mathrm{th}}$, соответственно; $a_{10}=v_{1 \mathrm{th}} / \eta$, где $\eta=\tau_{r} / \tau_{p h}, \tau_{r}$ и $\tau_{p h}=\left(v_{g} \alpha_{s}\right)^{-1}$ время жизни носителей и фотонов во внутреннем резонаторе $\left(v_{g}\right.$ - групповая скорость для излучения накачки и сигнала), $v_{1 \text { th }}-$ пороговое значение числа носителей, $T=2 \alpha_{s} L_{i n}-$ потери в резонаторе за проход, причем $\alpha_{s}=\alpha_{i n}-\left(L_{i n}\right)^{-1} \ln \left[r_{\mathrm{DBM}}\left(1-r^{2}\right) R\right], \alpha_{i n}-$ внутренние потери вследствие рассеяния и нерезонансного поглощения во внутреннем резонаторе, $r_{\mathrm{DBM}}, r$ и $R$ - коэффициенты отражения двухполосного БЗ, внешней поверхности активного зеркала и сферического выходного зеркала, соответственно. Нормированный коэффициент усиления $G_{1}$ принимается в форме

$$
G_{1}=1+G_{10} \ln v_{1}
$$

где $G_{10}=4 m G_{Q W} / T, m$ - число квантовых ям в активной области, $G_{Q W}$ коэффициент усиления, приходящийся на одну квантовую яму. Принимая во внимание эти параметры, пороговое значение числа носителей можно записать в виде $v_{1 \text { th }}=m \pi \omega_{p p}^{2} N_{t} \exp \left(1 / G_{10}\right)$, где $\omega_{p p}$ радиус пучка первичной оптической накачки, $N_{t}$ плотность числа носителей, соответствующая просветлению активной среды (transparent carrier density). Коэффициент нелинейного взаимодействия определяется в форме $\delta=\mu a_{10} / \tau_{i n}$, где $\tau_{i n}=2 L_{i n} / v_{g}$ - время обхода внутреннего резонатора, $\mu=2 \gamma /\left[\pi\left(w_{1}^{2}+w_{2}^{2}\right)\right]$. При выводе выражений предполагалось, что оптические поля накачки и сигнала представлены в форме гауссовых пучков с радиусами перетяжек внутри кристалла $w_{1}$ и $w_{2}$,

$$
\gamma=\frac{32 Z_{0}}{n_{1} n_{2} n_{3}} d_{14}^{2}\left(\frac{L_{c}^{2}}{\lambda_{1} \lambda_{2}}\right) \hbar \omega_{3}
$$

$Z_{0}=120 \pi$ - волновое сопротивление вакуума, $d_{14}$ - элемент тензора нелинейной восприимчивости кристалла GaAs, $L_{c}$ - длина кристалла, $\lambda_{1}$ и $\lambda_{2}$ - длина волны накачки и сигнальной волны, $n_{1,2,3}$ - коэффициенты преломления, $\hbar \omega_{3}$ - энергия фотонов холостой волны. В уравнениях (1) $\sigma$ соответствует мощности внешней первичной накачки $P$, нормированной на пороговое значение $P_{\text {th }}$. Точки над переменными означают дифференцирование по величине $t / \tau_{r}$. 


\section{2. Численные результаты}

Численный анализ динамики системы выполнялся с применением стандартных методов нелинейной динамики: построение временных реализаций, фазовых портретов, фурье-спектров. Бифуркационный анализ состояния равновесия системы проводился с помощью пакета DDEBiftool [18]. Также была выполнена проверка наличия хаоса, для чего вычислялся старший показатель Ляпунова. Необходимо отметить, что для изучения динамики системы были выбраны значения запаздывания $\tau$, нормированной мощности первичной накачки $\sigma / \sigma_{O P O}$ и длины кристалла $L_{c}$, которые отвечают возможности практической реализации. Результаты, полученные разными методами, хорошо согласуются между собой.

Ранее полученные результаты [15] показывают, что в случае внутрирезонаторной генерации разностной частоты $f$ для всех практически важных значений управляющих параметров состояние равновесия системы остается устойчивым. Другими словами, наблюдается генерация с постоянной амплитудой. Этот результат представляется важным с точки зрения использования таких генераторов в спектроскопии высокого разрешения.

В случае параметрической генерации ситуация сложнее. На плоскости управляющих параметров имеются области, где устойчивость состояния равновесия нарушается. На рис. 2 в виде $\mathrm{D}, \mathrm{D} \cup \mathrm{C}$ и $\mathrm{D} \cup \mathrm{C} \cup \mathrm{B}$ обозначены такие области. Каждая из областей отвечает определенному значению длины нелинейного кристалла $L_{c}$ при прочих фиксированных значениях параметров. При переходе через линии $l^{\mathrm{AB}}, l^{\mathrm{BC}}, l^{\mathrm{CD}}$ наблюдается потеря устойчивости для $L_{c}=7,6$ и 5 мм, соответственно. При построении графиков $\sigma$ нормировалась на значение $\sigma_{\mathrm{OPO}}=11.03$, соответствующее значению величины первичной накачки на пороге параметрической генерации при $L_{c}=5$ мм. При переходе через границы данных областей старшая пара комплексносопряженных корней характеристического уравнения приобретает положительную действительную часть, наблюдается супекритическая бифуркация Андронова-Хопфа. Однако область устойчивости возникающих периодических колебаний ограничена, при вариации управляющих параметров они быстро сменяются квазипериодическими колебаниями.

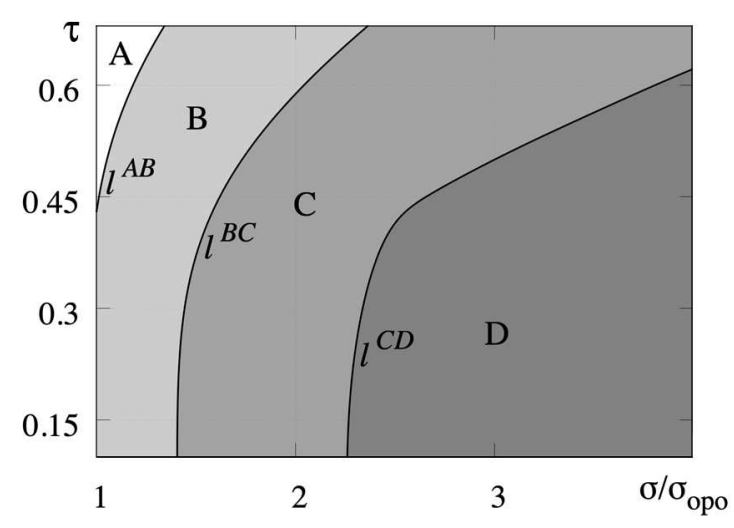

Рис. 2. Разбиение плоскости параметров $\sigma / \sigma_{\mathrm{OPO}}-\tau$ на области с различными типами устойчивости. Линии суперкритической бифуркации Андронова-Хопфа обозначены $l^{\mathrm{AB}}, l^{\mathrm{BC}}, l^{\mathrm{CD}}$. Область А отвечает устойчивой генерации для всех рассматриваемых значений длины кристалла. Области $\mathrm{D}, \mathrm{D} \cup \mathrm{C}$ и $\mathrm{D} \cup \mathrm{C} \cup \mathrm{B}$ соответствуют неустойчивости стационарного состояния системы при $L_{c}=5 ; L_{c}=6 ; L_{c}=7$ мм

Fig. 2. Areas on the parameter plain $\sigma / \sigma_{\mathrm{OPO}}-\tau$ with different types of stability. The lines of the supercritical AndronovHopf bifurcation are marked $l^{\mathrm{AB}}, l^{\mathrm{BC}}, l^{\mathrm{CD}}$. Region A corresponds to stable generation for all considered values of the crystal length. The areas $\mathrm{D}, \mathrm{D} \cup \mathrm{C}$ and $\mathrm{D} \cup \mathrm{C} \cup \mathrm{B}$ correspond to the instability of the steady state of the system for $L_{c}=5 \mathrm{~mm}$, $L_{c}=6 \mathrm{~mm}, L_{c}=7 \mathrm{~mm}$ 
Для иллюстрации на рис. 3 и 4 приведены характерные колебательные режимы излучения для различных значений нормированной мощности первичной накачки $\sigma / \sigma_{O P O}$ при фиксированном времени запаздывания $\tau=0.1$ и длине кристалла $L_{c}=5$ мм. Для малых значений $\sigma$, то есть за пределами области $\mathrm{D}$ на рис. 2 , после завершения переходного процесса устанавливается режим стационарной генерации (см. рис. 3). Переход в заштрихованную область D сопровождается возбуждением периодических колебаний амплитуды на частоте $f=14 \pi / \tau$, что соответствует седьмой гармонике межмодовых биений для рассматриваемого резонатора или 35 ГГц. Необходимо отметить, что область устойчивости периодических колебаний находится в непосредственной близости от бифуркационной линии стационарного состояния. Так, для $\tau=0.1$ бифуркация Андронова-Хопфа происходит при $\sigma / \sigma_{\mathrm{OPO}} \approx 2.250$, а потеря устойчивости периодических колебаний - при $\sigma / \sigma_{\mathrm{OPO}} \approx 2.257$. Бифуркационный анализ показывает, что характер развивающейся неустойчивости меняется на квазипериодический в результате бифуркации Неймарка-Сакера, в фазовом пространстве системы возникает устойчивый двухчастотный тор (см. рис. 4).

Для подтверждения вывода о квазипериодическом характере динамики системы в области неустойчивости состояния равновесия был вычислен старший показатель Ляпунова. Применявшаяся для этого стандартная процедура состоит в том, что сначала для изучаемой системы выводится вариационное уравнение, представляющее собой линеаризацию исходной системы вдоль траектории и описывающее эволюцию вектора инфинитезимального возмущения этой траектории. Так как вариационное уравнение линейно по отношению к вектору возмущения, норма этого вектора растёт или убывает в среднем по закону экспоненты, показатель которой называется показателем Ляпунова. Для вычисления этого показателя нужно решать вариационное уравнение совместно с исходной системой, периодически выполняя перенормировку вектора возмущения. При этом логарифмы накопленных к моменту очередной перенормировки норм следует суммировать. Тогда, пройдя достаточно протяжённый отрезок траектории и усреднив накопленные логарифмы норм по времени, мы получим старший показатель Ляпунова. Отметим, что при вычислении более одного показателя Ляпунова применяют алгоритм, идея которого впервые была предложена независимо Бенеттином с соавторами [19] и Шимадой и Нагашимой [20]. Подробное описание этого алгоритма, включающего наряду с перенормировками также ортогонализации векторов возмущений, можно найти, например, в статьях $[21,22]$.
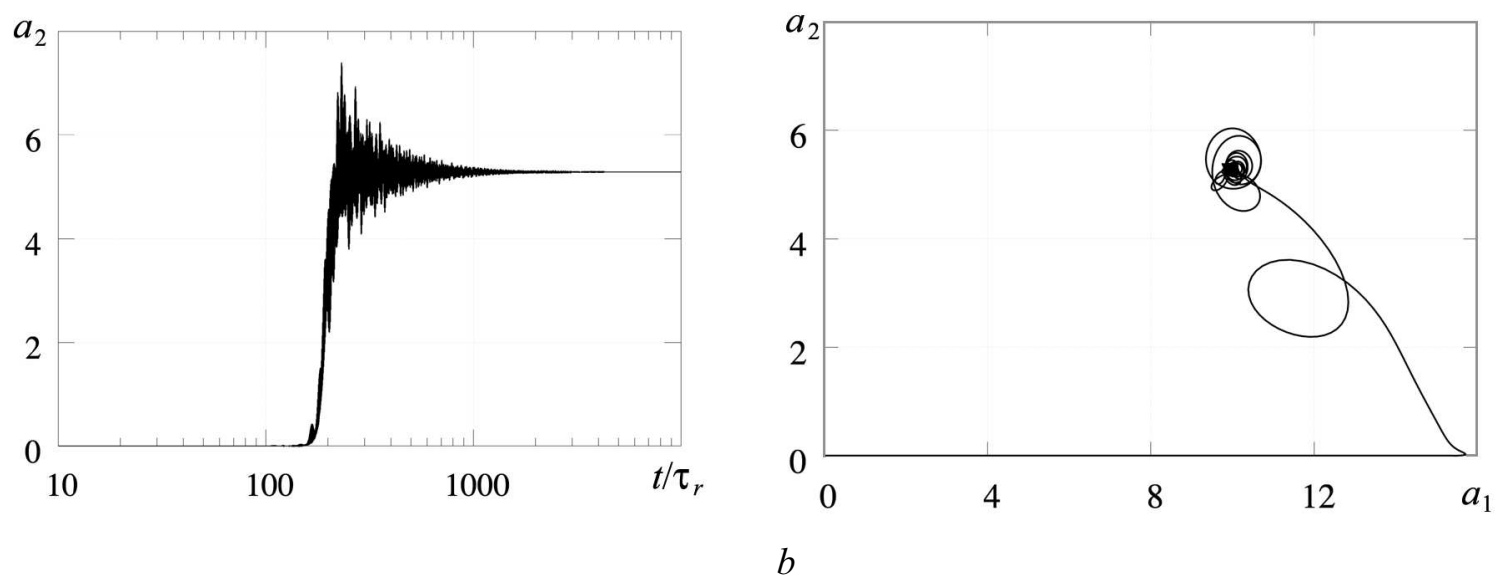

Рис. 3. Устойчивый режим генерации при $\sigma / \sigma_{\mathrm{OPO}}=1.5$. Показаны $(a)$ временная реализация сигнального излучения и $(b)$ фазовый портрет в координатах $\left(a_{1}, a_{2}\right)$

Fig. 3. Stable generation regime for $\sigma / \sigma_{\mathrm{OPO}}=1.5$. Time series $(a)$ of signal radiation and $(b)$ phase portrait in coordinates $\left(a_{1}, a_{2}\right)$ are shown 

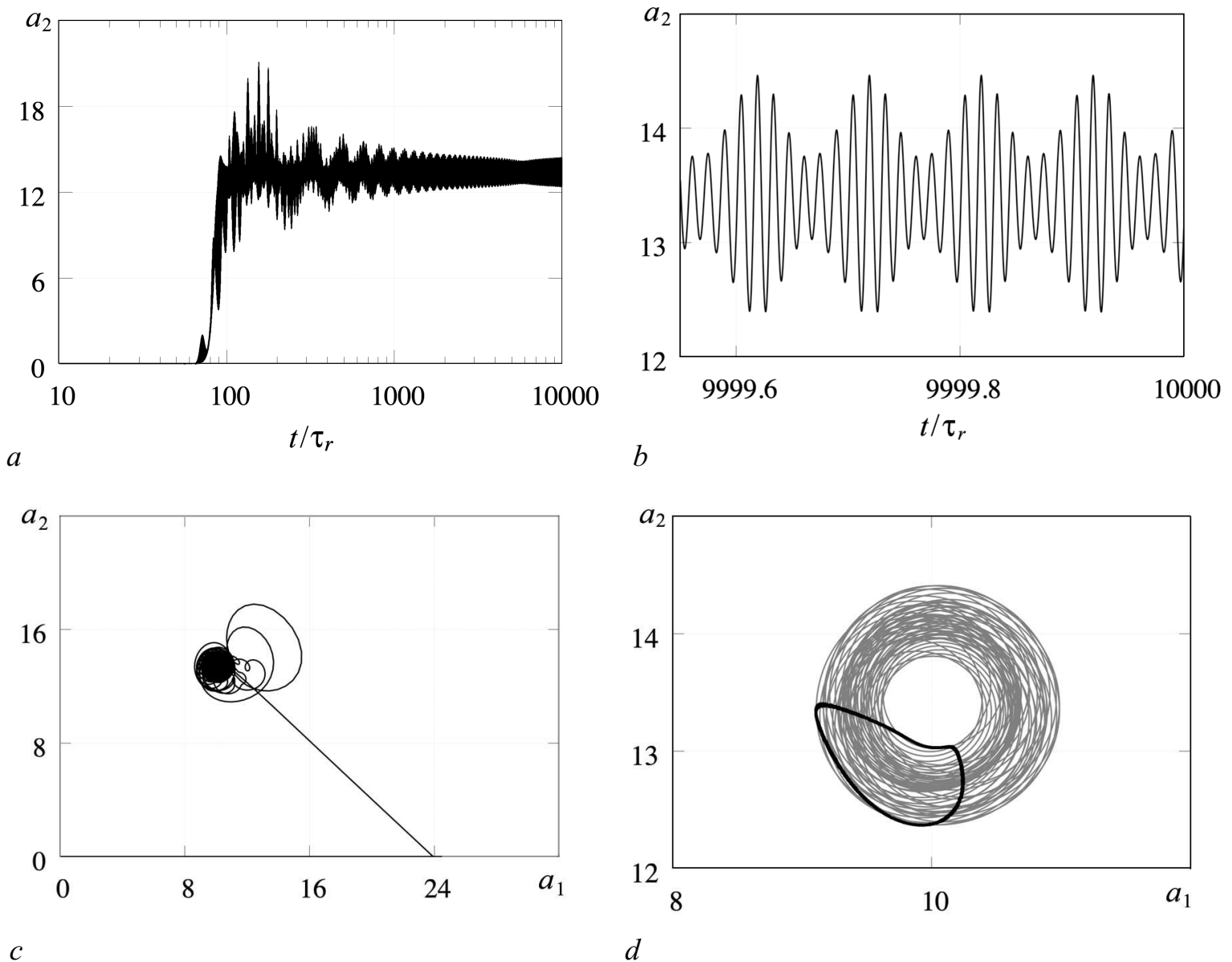

$c$

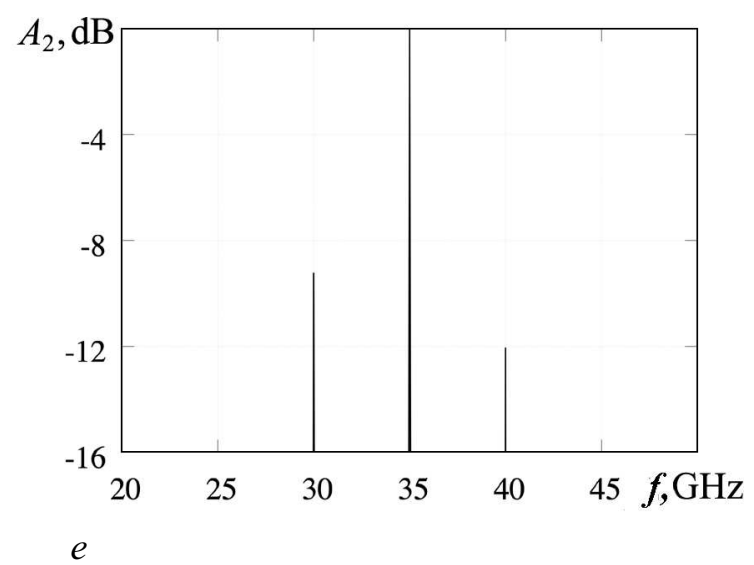

Рис. 4. Квазипериодические колебания амплитуды при $\sigma / \sigma_{\mathrm{OPO}}=2.26$. Показаны временные реализации $(a, b)$ и фазовые портреты переходного процесса $(c)$ и установившегося режима $(d)$ (черным цветом показано сечение Пуанкаре); $e$ - амплитудный спектр установившегося режима колебаний

Fig. 4. Quasi-periodic oscillations of the amplitude for $\sigma / \sigma_{\mathrm{OPO}}=2.26$. Time series $(a, b)$ and phase portraits the transition process $(c)$ and the steady state regime $(d)$ (black limit cycle denotes the Poincare section); $e$ - the amplitude spectrum of the stationary regime

Кочкуров Л.А., Балакин М.И., Купџов П.В., Морозов Ю.А.

Известия вузов. ПНД, 2019, т. 27, № 3 
Уравнение в вариациях для системы уравнений (1) имеет следующий вид:

$$
\left(\begin{array}{c}
\dot{\tilde{a}}_{1} \\
\dot{\tilde{a}}_{2} \\
\dot{\tilde{v}}_{1}
\end{array}\right)=\frac{\partial F\left(a_{1}, a_{2}, v_{1}, a_{1 \tau}, a_{2 \tau}\right)}{\partial\left(a_{1}, a_{2}, v_{1}\right)}\left(\begin{array}{c}
\tilde{a}_{1} \\
\tilde{a}_{2} \\
\tilde{v}_{1}
\end{array}\right)+\frac{\partial F\left(a_{1}, a_{2}, v_{1}, a_{1 \tau}, a_{2 \tau}\right)}{\partial\left(a_{1 \tau}, a_{2 \tau}\right)}\left(\begin{array}{c}
\tilde{a}_{1 \tau} \\
\tilde{a}_{2 \tau} \\
0
\end{array}\right) .
$$

Здесь $F\left(a_{1}, a_{2}, v_{1}, a_{1 \tau}, a_{2 \tau}\right)$ - правая часть системы (1), а $\partial F\left(a_{1}, a_{2}, v_{1}, a_{1 \tau}, a_{2 \tau}\right) / \partial\left(a_{1}, a_{2}, v_{1}\right)$ и $\partial F\left(a_{1}, a_{2}, v_{1}, a_{1 \tau}, a_{2 \tau}\right) / \partial\left(a_{1 \tau}, a_{2 \tau}\right)$ обозначают матрицы производных (матрицы Якоби), вычисленные по отношению к переменным $a_{1}, a_{2}, v_{1}$ и запаздывающим переменным $a_{1 \tau}, a_{2 \tau}$, соответственно. Переменные с тильдой $\tilde{a}_{1}, \tilde{a}_{2}$ и $\tilde{v}_{1}$ обозначают возмущения траектории, которые мы вычисляем. С формальной точки зрения система с запаздыванием (1) имеет бесконечно большую размерность фазового пространства, а её вектор возмущения представляет собой объединение двух отрезков траектории $\left[\tilde{a}_{1}(t-\tau), \tilde{a}_{1}(t)\right],\left[\tilde{a}_{2}(t-\tau), \tilde{a}_{2}(t)\right]$ и текущего значения переменной $\tilde{v}_{1}(t)$, для которой нет запаздывания. При численном счёте дискретизация по времени одновременно задаёт дискретизацию вектора возмущения так, что его размерность равна $2(\tau / h+1)+1$, где $h$ - шаг численного счёта. Более подробное обсуждение теории и численных методов ляпуновского анализа систем с запаздыванием можно найти, например, в работах $[23,24]$. Для системы (1) мы вычислили старший показатель Ляпунова для восьми произвольно выбранных точек внутри заштрихованной области $D$ на рис. 2 , а именно для $\sigma / \sigma_{O P O}=2.72$ и 3.17 для запаздываний $\tau=0.1,0.2,0.3,0.4$. Вычисления проводились с постоянным шагом $h=10^{-5}$. Во всех случаях показатель попал в диапазон от $-7 \cdot 10^{-4}$ до $2 \cdot 10^{-4}$, то есть оказался достаточно малым по абсолютной величине. Это даёт основания полагать, что фактическое значение старшего показателя Ляпунова во всех восьми рассмотренных случаях равно нулю, а отклонения полученных значений от нуля следует считать численной погрешностью. Таким образом, анализ показателей Ляпунова подтверждает сделанный выше вывод о квазипериодическом характере динамики изучаемой системы при рассматриваемых значениях параметров.

\section{Заключение}

В работе представлена модель внутрирезонаторного оптического параметрического генератора с внутрирезонаторной накачкой полупроводниковым дисковым лазером. Исследование динамики системы проведено с использованием средств численного интегрирования, бифуркационного анализа систем с временным запаздыванием и расчета спектра показателей Ляпунова. Показано, что, в отличие от генератора разностной частоты, вариация управляющих параметров в исследуемой системе может приводить к потере устойчивости состояния равновесия, соответствующего стационарной генерации. Развивающаяся неустойчивость носит квазипериодический характер для всех рассмотренных случаев, лежащих в пределах физической реализуемости параметров устройства. Предметом дальнейших исследований будет изучение причин возникновения неустойчивости, а также изучение характерных бифуркационных переходов, в том числе приводящих к мультистабильности.

\section{Библиографический список}

1. Sorokina I.T., Vodopyanov K.L., eds. Solid-State Mid-Infrared Laser Sources. Ser. Topics in Applied Physics, Vol. 89. Springer-Verlag, 2003. 558 p.

2. Morozov Y.A., Morozov M.Y., Kozlovsky V.I., Okhotnikov O.G. Compact intracavity singly-resonant optical parametric oscillator pumped by GaSb-based vertical external cavity surface-emitting laser: Concept and the main operational characteristics // IEEE J. of Selected Topics in Quantum Electron. 2015.Vol. 21, no. 1. 1603105-1-1603105-5. 
3. Morozov Y.A. Multi-mode dynamics of optical oscilators based on intracavity nonlinear frequency down-conversion // Appl. Phys B. 2018. Vol. 124, no. 1. 12-1-12-7.

4. Stothard D.J.M., Hopkins J.-M., Burns D., Dunn M.H. Stable, continuous-wave, intracavity, optical parametric oscillator pumped by a semiconductor disk laser (VECSEL) // Optics Express. 2009. Vol. 17, no. 13. P. 10648-10658.

5. Siegman A.E. Lasers, University Science Book, 20 Edgehill Road, Mill Valley, California, 1986.

6. Oshman M.K. and Harris S.E. Theory of optical parametric oscillation internal to the laser cavity // IEEE J. Quantum Electron. 1968. Vol. QE-4, no. 8. P. 491-502.

7. Hodges S.E., Munroe M., Cooper J., Raymer M.G. Multimode laser model with coupled cavities and quantum noise // JOSA B. 1997. Vol. 14, no. 1. P. 191-199.

8. Turnbull G.A., Dunn M.H., Ebrahimzadeh M. Continuous-wave, intracavity optical parametric oscillators: an analysis of power characteristics // Appl. Phys B. 1998. Vol. 66. P. 701-710.

9. Morozov Y.A. Transient power characteristics of a compact singly resonant intracavity optical parametric oscillator pumped by a semiconductor disk laser // JOSA B. 2016. Vol. 33, no. 7. P. $1470-1475$.

10. Park J.-D., Seo D.-S., McInerney J. Self-pulsations in strongly coupled asymmetric external cavity semiconductor lasers // IEEE J. Quantum Electron. 1990. Vol. 26, no. 8. P. 1353-1362.

11. Hui R.-Q., Tao S.-P. Improved rate equations for external cavity semiconductor lasers // IEEE J. Quantum Electron. 1989. Vol. 25. P. 1580-1584.

12. van Tartwijk G.H. M., Lenstra D. Semiconductor laser with optical injection and feedback // Quantum Semiclass. Opt. 1995. Vol. 7. P. 87-143.

13. Morozov Y.A., Leinonen T., Härkönen A., Pessa M. Simultaneous dual-wavelength emission from vertical external-cavity surface-emitting laser: A numerical modeling // IEEE J. Quantum Electron. 2006. Vol. 42. P. 1055-1061.

14. Lang R., Kobayashi K. External optical feedback effects on semiconductor injection laser properties // IEEE J. Quantum Electron. 1980. Vol. 16. P. 347-355.

15. Морозов Ю.А., Балакин М.И., Кочкуров Л.А., Морозов М.Ю. Генератор разностной частоты и оптический параметрический генератор с внутрирезонаторной накачкой полупроводниковым дисковым лазером: Сопоставительный анализ в модели с запаздыванием // Изв. Сарат. ун-та. Нов. сер. Сер. Физика. 2019. Т. 19, № 1. С. $34-42$.

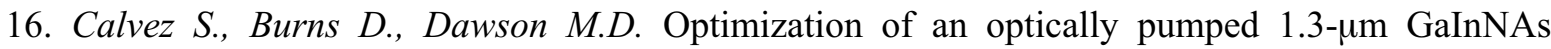
vertical-cavity surface-emitting laser // IEEE Phot. Techn. Lett. 2002. Vol. 14, no. 2. P. 131-133.

17. Tropper A.C., Hoogland S. Extended cavity surface-emitting semiconductor lasers // Prog. Quantum Electronics. 2006. Vol. 30. P. 1-43.

18. Engelborghs K., Luzyanina T., Samaey G. DDE-BIFTOOL. v.2.00 user manual: a Matlab package for bifurcation analysis of delay differential equations. Technical Report TW-330, Katholieke Universiteit Leuven, Leuven, Belgium (2001).

19. Benettin G., Galgani L., Giorgilli A., Strelcyn J.-M. Lyapunov characteristic exponents for smooth dynamical systems and for Hamiltonian systems: A method for computing all of them. Part 1: Theory // Meccanica. 1980. Vol. 15, no. 1. P. 9-20.

20. Shimada I., Nagashima T. A numerical approach to ergodic problem of dissipative dynamical systems // Prog. Theor. Phys. 1979. Vol. 61, no. 6. P. 1605-1616.

21. Kuptsov P., Parlitz U. Theory and computation of covariant Lyapunov vectors // Journal of Nonlinear Science. 2012. Vol. 22, no. 5. P. 727-762. DOI 10.1007/s00332-012-9126-5.

22. Купщов П.В. Вычисление показателей Ляпунова для распределенных систем: Преимуще- 
ства и недостатки различных численных методов // Изв. вузов. Прикладная нелинейная динамика. 2010. Т. 18, № 5. С. 93-112.

23. Kuptsov P.V., Kuznetsov S.P. Numerical test for hyperbolicity of chaotic dynamics in time-delay systems // Phys. Rev. E. 2016. Vol. 94. 010201(R).

24. Kuptsov P.V., Kuznetsov S.P. Numerical test for hyperbolicity in chaotic systems with multiple time delays // Communications in Nonlinear Science and Numerical Simulation. 2018. Vol. 56. P. 227-239.

\section{References}

1. Sorokina I.T., Vodopyanov K.L., eds. Solid-State Mid-Infrared Laser Sources. Ser. Topics in Applied Physics, Vol. 89, Springer-Verlag, 2003, 558 p.

2. Morozov Y.A., Morozov M.Y., Kozlovsky V.I., Okhotnikov O.G. Compact intracavity singlyresonant optical parametric oscillator pumped by GaSb-based vertical external cavity surfaceemitting laser: Concept and the main operational characteristics, IEEE J. of Selected Topics in Quantum Electron, 2015, Vol. 21, no. 1, 1603105-1-1603105-5.

3. Morozov Y.A. Multi-mode dynamics of optical oscilators based on intracavity nonlinear frequency down-conversion. Appl. Phys B, 2018, vol. 124, no. 1, 12-1-12-7.

4. Stothard D.J.M., Hopkins J.-M., Burns D., Dunn M.H. Stable, continuous-wave, intracavity, optical parametric oscillator pumped by a semiconductor disk laser (VECSEL). Optics Express, 2009, vol. 17, no. 13, pp. 10648-10658.

5. Siegman A.E. Lasers, University Science Book, 20 Edgehill Road, Mill Valley, California, 1986.

6. Oshman M.K. and Harris S.E. Theory of optical parametric oscillation internal to the laser cavity. IEEE J. Quantum Electron., 1968, Vol. QE-4, no. 8. pp. 491-502.

7. Hodges S.E., Munroe M., Cooper J., Raymer M.G. Multimode laser model with coupled cavities and quantum noise. JOSA B, 1997, vol. 14, no. 1, pp. 191-199.

8. Turnbull G.A., Dunn M.H., Ebrahimzadeh M. Continuous-wave, intracavity optical parametric oscillators: An analysis of power characteristics. Appl. Phys B, 1998, vol. 66, pp. 701-710.

9. Morozov Y.A. Transient power characteristics of a compact singly resonant intracavity optical parametric oscillator pumped by a semiconductor disk laser. JOSA B, 2016, vol. 33, no. 7, pp. 1470-1475.

10. Park J.-D., Seo D.-S., McInerney J. Self-pulsations in strongly coupled asymmetric external cavity semiconductor lasers. IEEE J, Quantum Electron., 1990, vol. 26, no. 8, v. 1353-1362.

11. Hui R.-Q., Tao S.-P. Improved rate equations for external cavity semiconductor lasers. IEEE $J$. Quantum Electron., 1989, vol. 25. pp. 1580-1584.

12. van Tartwijk G.H. M., Lenstra D. Semiconductor laser with optical injection and feedback. Quantum Semiclass. Opt., 1995, vol. 7, pp. 87-143.

13. Morozov Y.A., Leinonen T., Härkönen A., Pessa M. Simultaneous dual-wavelength emission from vertical external-cavity surface-emitting laser: A numerical modeling. IEEE J. Quantum Electron., 2006, vol. 42, pp. 1055-1061.

14. Lang R., Kobayashi K. External optical feedback effects on semiconductor injection laser properties. IEEE J. Quantum Electron., 1980, vol. 16, pp. 347-355.

15. Morozov Y.A., Balakin M.I., Kochkurov L.A., Morozov M.Y. Generator difference frequency and optical parametric generator with intracavity-pumped semiconductor disk laser: A comparative analysis of the models with a delay. Izv. of Saratov State University. New Ser. Ser. Phyzika, 2019, vol. 19, no. 1, pp. 34-42 (in Russian). 
16. Calvez S., Burns D., Dawson M.D. Optimization of an optically pumped 1.3- $\mu$ m GaInNAs vertical-cavity surface-emitting laser. IEEE Phot. Techn. Lett. 2002, vol. 14, no. 2, pp. 131-133.

17. Tropper A.C., Hoogland S. Extended cavity surface-emitting semiconductor lasers, Prog. Quantum Electronics, 2006, vol. 30, pp. 1-43.

18. Engelborghs K., Luzyanina T., Samaey G. DDE-BIFTOOL. v.2.00 user manual: a Matlab Package for Bifurcation Analysis of Delay Differential Equations. Technical Report TW-330, Katholieke Universiteit Leuven, Leuven, Belgium (2001).

19. Benettin G., Galgani L., Giorgilli A., Strelcyn J.-M. Lyapunov characteristic exponents for smooth dynamical systems and for Hamiltonian systems: A method for computing all of them. Part 1: Theory. Meccanica, 1980, vol. 15, no. 1, pp. 9-20.

20. Shimada I., Nagashima T. A numerical approach to ergodic problem of dissipative dynamical systems. Prog. Theor. Phys., 1979, vol. 61, no. 6, pp. 1605-1616.

21. Kuptsov P., Parlitz U. Theory and computation of covariant Lyapunov vectors. Journal of Nonlinear Science, 2012, vol. 22, no. 5, pp. 727-762, DOI 10.1007/s00332-012-9126-5.

22. Kuptsov P.V. Computation of Lyapunov exponents for spatially extended systems: Advantages and limitations of various numerical methods. Izvestiya VUZ. Applied Nonlinear Dynamics, 2010, vol. 18, no. 5, pp. 93-112 (in Russian).

23. Kuptsov P.V., Kuznetsov S.P. Numerical test for hyperbolicity of chaotic dynamics in time-delay systems. Phys. Rev. E. 2016. Vol. 94. 010201(R).

24. Kuptsov P.V., Kuznetsov S.P. Numerical test for hyperbolicity in chaotic systems with multiple time delays. Communications in Nonlinear Science and Numerical Simulation, 2018, vol. 56, pp. $227-239$.

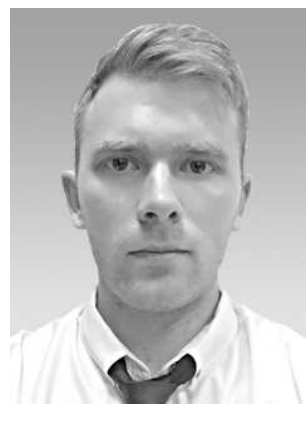

Кочкуров Леонид Алексеевич. Окончил Саратовский государственный университет (2012). Кандидат физико-математических наук (2017), доцент кафедры «Радиоэлектроника и телекоммуникации» Саратовского государственного технического университета имени Гагарина Ю.А. Научные интересы - лазерная физика, волоконная оптика, нелинейная оптика. Автор более 30 научных публикаций.

Россия, 410054 Саратов, ул. Политехническая, 77

Саратовский государственный технический университет имени Гагарина Ю.А.

E-mail: 1kochkurov@gmail.com

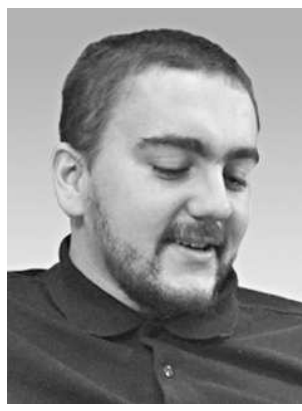

Балакин Максим Игоревич. Окончил Саратовский государственный университет (2011). Кандидат физико-математических наук (2015), доцент кафедры «Радиоэлектроника и телекоммуникации» Саратовского государственного технического университета имени Гагарина Ю.А. Научные интересы - нелинейная динамика, теория колебаний, системы с временным запаздыванием и мультистабильность. Автор более 30 научных публикаций.

Россия, 410054 Саратов, ул. Политехническая, 77

Саратовский государственный технический университет имени Гагарина Ю.А.

E-mail: balakinmaxim@gmail.com 

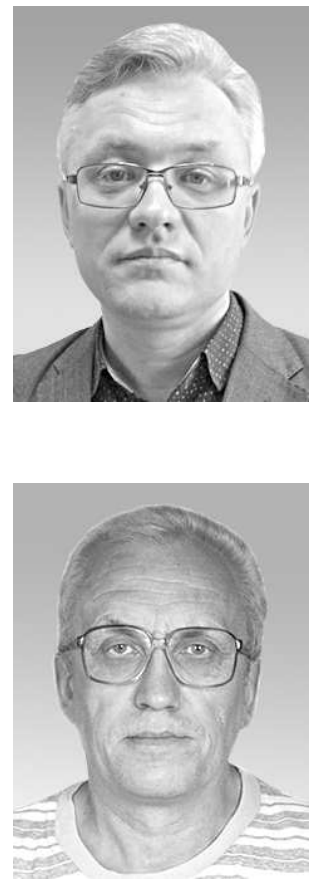

Купиов Павел Владимирович - профессор кафедры «Приборостроение» Саратовского государственного технического университета имени Гагарина Ю.А., а также сотрудник научной группы теоретической нелинейной динамики в Саратовском филиале Института радиотехники и электроники РАН. Область научных интересов - сложные колебания в распределённых системах, хаотическая динамика в системах высокой размерности, численные методы.

Россия, 410054 Саратов, ул. Политехническая, 77

Саратовский государственный технический университет имени Гагарина Ю.А.

E-mail: p.kuptsov@rambler.ru

Морозов Юрий Александрович - кандидат физико-математических наук, ведущий научный сотрудник Института радиотехники и электроники им. В.А. Котельникова Российской академии наук.

Россия, 410019 Саратов, ул. Зеленая, 38

Саратовский филиал Института радиотехники и электроники им. В.А. Котельникова РАН E-mail: yuri.mor@rambler.ru 